\title{
Effect of Specimen Shape on Test Results of Au Freestanding Film Measured by Strip Bending Method
}

\author{
Jungmin Park, Jae-Hyun Kim*, Sang-Joo Lee, \\ Bongkyun Jang, Bung-Ik Choi and Hak-Joo Lee \\ Korea Institute of Machinery and Materials, \\ 104 Sinseongno, Yuseong-gu, Daejeon 305-343, Korea
}

(Received June 22, 2009; accepted August 6, 2009)

Key words: $\quad$ strip bending test, specimen shape, $\mathrm{Au}$, stress-strain curve, finite element analysis

Strip bending is a simple and productive method for the mechanical characterization of thin-film materials used in micro-electromechanical systems (MEMS) applications. This method can yield a stress-strain curve as does a microtensile test, and can also yield the elastic modulus and strength. The strip bending test is much more useful than the microtensile test because of the ease of specimen fabrication, alignment, and handling. On the other hand, results obtained by the strip bending test can suffer from uncertainties related to specimen shape, and the specimen shape can affect the strain estimation and stress concentration of the specimen. In this study, two types of specimen were fabricated using a MEMS process to investigate the effect of specimen shape. Au was chosen as a thin-film material owing to its widespread use in MEMS applications. The stress-strain curves of $\mathrm{Au}$ thin film were measured using a strip bending apparatus equipped with an accurate laser displacement sensor and a load cell of $500 \mathrm{mN}$ capacity. The difference caused by the specimen shape was clearly identified in the stress-strain curves of the two types of specimen of the same Au thin film. A carefully designed microtensile test was performed to accurately measure the stress-strain curve of the Au thin film, and the tensile data was compared with the data obtained by the strip bending method. To understand the mechanics of the strip bending method and the effect of specimen shape, finite element analysis (FEA) was performed for 3-dimensional models of the two types of strip bending specimen. A desirable test practice of the strip bending method was suggested for standardization purposes on the basis of experimental and FEA results.

${ }^{*}$ Corresponding author: e-mail: jaehkim@kimm.re.kr 


\section{Introduction}

The mechanical properties of materials are basic information for designing products. ${ }^{(1,2)}$ For macroscale products, many standard methods of measuring the mechanical properties of macroscale specimens have been developed. As micromachining technology improves, microscale products become more popular and the need to characterize microscale structures increases. ${ }^{(1)}$ Since the mechanical properties of microscale structures are different from those of bulk-scale structures and are strongly dependent on the fabrication process, ${ }^{(2)}$ it is necessary to measure these properties using specimens with the same characteristic scales and fabricated by the same process. The mechanical characterization of microscale structures is an exciting topic, and many researchers have tried to develop accurate and easy methods for such characterization. ${ }^{(3)}$

The strip bending method is one of the most promising methods for measuring the mechanical properties of freestanding thin films since it can yield stress-strain data of microscale structures with moderate effort. The best method for measuring stressstrain data is the microtensile test, which is a counterpart of the macroscale tensile test for microscale structures. In the microtensile test, the stress and strain distributions are uniform along the gauge section of the specimen, which yields excellent stress-strain data. However, accurate microtensile data are difficult to obtain using this method owing to obstacles such as specimen gripping, alignment, load, and strain measurement. ${ }^{(3,4)}$ The strip bending method can be a good substitute for the difficult microtensile test because of its usefulness and simplicity, ${ }^{(5-9)}$ and can be attractive to industries for microscale devices such as flexible electronic devices, micro-electromechanical systems (MEMS), and nanoelectromechanical systems (NEMS). The data obtained by the strip bending method can include some errors caused by specimen shape, even with excellent alignment and loaddisplacement measurement. The motivation for this study is our desire to understand the errors induced by the specimen shape, to find a method of minimizing such errors, and to demonstrate the simplicity and usefulness of the strip bending test, which can provide accurate stress-strain data similarly to the microtensile test.

The objective of this study is to determine the effect of specimen shape when performing the strip bending method and to minimize the error caused by specimen shape. To this end, we start by briefly describing the principle of the strip bending method. Experimental details of the strip bending test are presented including those of the fabrication of the specimens, apparatus, and test procedure. The results obtained are presented and compared with the results obtained by the microtensile test and finite element analysis. Our findings and explanations are given, and a desirable test practice is suggested for the standardization of the strip bending method. Finally, a summary is presented.

\section{Strip Bending Method}

\subsection{Principle of strip bending method}

A schematic view of the strip bending test is shown in Fig. 1(a). The loading tip moves in the $z$-direction and applies a force to the strip that can be regarded as a bridge 
(a)

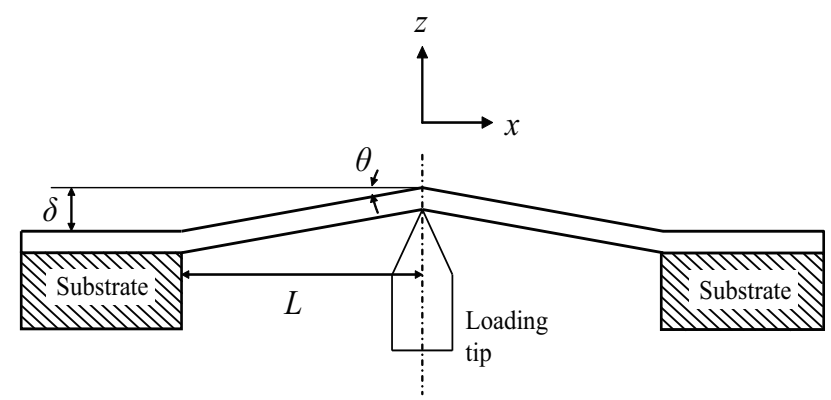

(b)

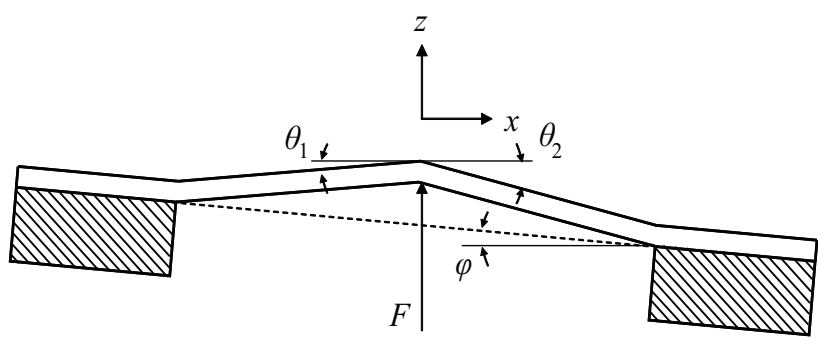

Fig. 1. Schematic of strip bending method.

connecting two fixed ends. The load and corresponding displacement are measured simultaneously during the movement. The stress-strain curve of the strip is estimated from the measured load and displacement using the analysis model described in the next section. When the alignment between the strip and the loading tip is poor as shown in Fig. 1(b), the analysis model should be corrected to yield a good estimation of stress and strain, as will be described in $\S 2.3$.

\subsection{Two types of specimen}

Two types of specimen are considered in this study. As shown in Fig. 2(a), one is a straight strip, and the other is a curved strip. Since the strain distribution in the straight strip is nearly uniform except in the fixed end region and loading region, it is easier to estimate strain for the straight strip than for the curved strip, where the strain distribution is nonuniform and complex. On the other hand, the straight strip is more sensitive to stress concentration than the curved strip, and thus leads to an underestimation of the strength. The fabricated specimens are also shown in Fig. 2(b), and their fabrication process is described in $\S 3.1$. It is possible to fabricate many strip specimens (regardless of specimen shape) on a single die of substrate, and the strip bending method can be easily automated to test each strip specimen sequentially using a motorized specimen holder as in refs. $6-8$. 


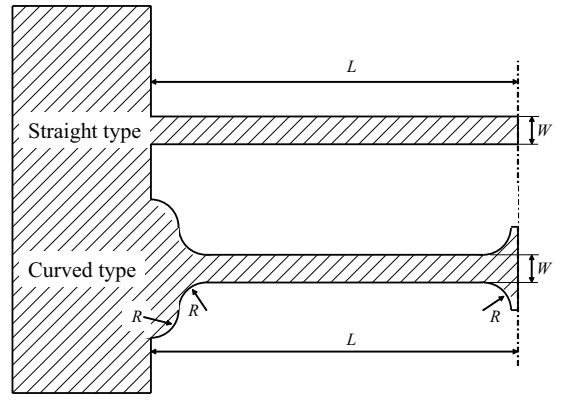

(a)
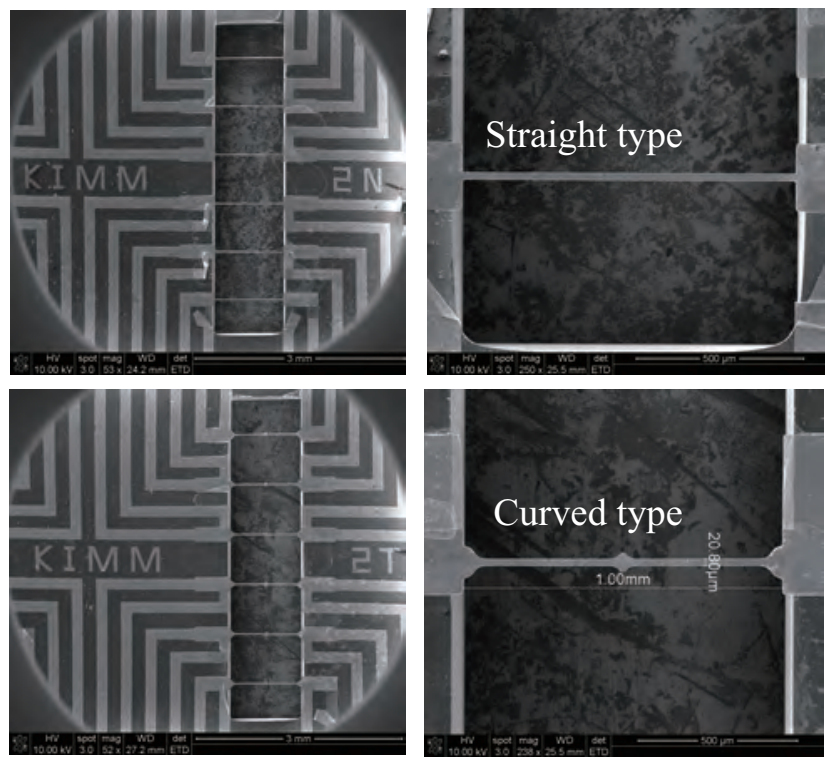

(b)

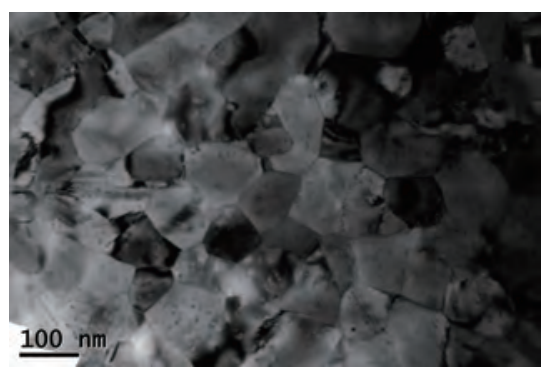

(c)

Fig. 2. Two types of strip specimen. (a) Shape and physical dimensions $(L=500 \mu \mathrm{m}, W=20$ $\mu \mathrm{m}, R=40 \mu \mathrm{m}$ ). (b) SEM images of fabricated specimens. (c) A TEM image of a fabricated specimen. 


\subsection{Analysis of strip bending test}

For a strip with a stringlike behavior, axial stress and strain can be related to load and displacement via the following equations without any assumption on its constitutive law: $(5,6)$

$$
\begin{gathered}
\sigma=\frac{F}{2 B h \sin \theta}, \\
\varepsilon=\frac{\sqrt{L^{2}+\delta^{2}}}{L}-1=\frac{1-\cos \theta}{\cos \theta},
\end{gathered}
$$

where $\theta=\tan ^{-1}(\delta / L)$. Equation (1) is obtained from the static equilibrium of the strip under a force $F$ assuming that the strip cannot sustain a bending moment. Equation (2) is obtained by assuming that the axial strain is uniform along the entire length of the strip. Note that the stress concentrations at the fixed ends and in the middle of the strip are neglected under this assumption. The assumptions required to derive eqs. (1) and (2) can lead to errors, and these should be minimized to obtain accurate results from the strip bending test.

When the specimen is misaligned in the direction perpendicular to the loading tip as shown in Fig. 1(b), it will deform unsymmetrically. Since eqs. (1) and (2) are derived under the assumption of symmetric deformation, these equations lead to errors when the deflection angles of both sides of the specimen are not identical. When one can simultaneously measure the deflection angles of both sides of the specimen, eqs. (1) and (2) should be modified to eqs. (3) and (4), respectively. These are reduced to eqs. (1) and (2) when $\theta_{1}=\theta_{2}$ and $\varphi=0$.

$$
\begin{gathered}
\sigma=\frac{F}{B h\left(\sin \theta_{1}+\sin \theta_{2}\right)} \\
\varepsilon=\left(\frac{\csc \left(\theta_{1}-\varphi\right)+\csc \left(\theta_{2}-\varphi\right)}{\cot \left(\theta_{1}-\varphi\right)+\cot \left(\theta_{2}-\varphi\right)}\right)-1
\end{gathered}
$$

\section{Experiments}

\subsection{Fabrication of specimens}

The fabrication process for the specimens is summarized in Fig. 3. A 6-inch silicon wafer with an oxide layer was used as the substrate, as shown in Fig. 3(a). A thin film $(1 \mu \mathrm{m})$ of $\mathrm{Au}$ was sputter-deposited on the substrate after the deposition of a $\mathrm{Cr}$ adhesion layer of $10 \mathrm{~nm}$ thickness, as shown in Fig. 3(b). Strip specimens were defined and patterned by optical lithography, as shown in Fig. 3(c). Using deep reactive ion etching (DRIE), the backside silicon was etched out to release the specimen in freestanding form, as shown in Fig. 3(d). The oxide layer was etched as shown in Fig. 3(e), and then the Cr layer was etched, as shown in Fig. 3(f). The average grain size of the Au thin film was 


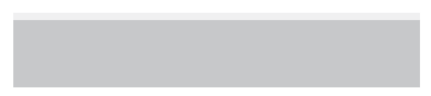

(a) Oxidation $\left(\mathrm{SiO}_{2}, 1000 \mathrm{~nm}\right)$

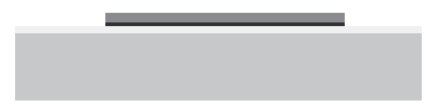

(c) Optical lithography

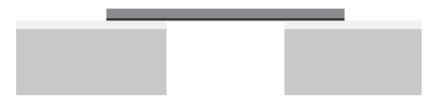

(e) Release (HF)

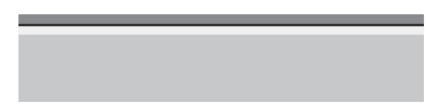

(b) Deposition (Cr, $10 \mathrm{~nm}$ and $\mathrm{Au}, 1000 \mathrm{~nm})$

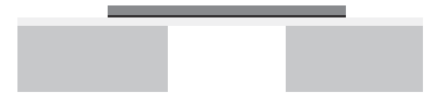

(d) DRIE Etching

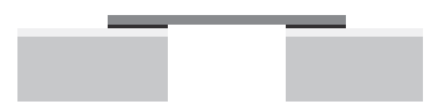

(f) Release (Cr etchant)

Fig. 3. Fabrication procedure.

determined to be $98.2 \mathrm{~nm}$ using transmission electron microscopy (TEM), as shown in Fig. 2(c).

\subsection{Strip bending apparatus}

The strip bending apparatus was developed in this study and is shown in Fig. 4. This apparatus consisted of a specimen holder with a five-axis adjustment stage for alignment, a load cell (LTS-50GA, Kyowa) with a $500 \mathrm{mN}$ capacity, a laser displacement sensor (LT-9000, Keyence) with a $10 \mathrm{~nm}$ resolution, a motorized translation stage with a minimum translation resolution of $50 \mathrm{~nm}$, and a wedge-shaped loading tip. The wedge shape was adopted because such a shape enables line contact with a strip specimen. A tip made of diamond was used to minimize tip deformation during testing. The tip was machined to a radius of $0.91 \mu \mathrm{m}$, as shown in Fig. 4. The loading tip was attached to a motorized translation stage, and moved vertically during the test. The load and displacement of the specimen were simultaneously measured using a load cell and a laser displacement sensor, respectively. A stress-strain curve was derived from these load and displacement data using the analysis model described previously. A laser displacement sensor was chosen since it could measure the vertical deflection of the specimen with a resolution of $10 \mathrm{~nm}$ at $2 \mu \mathrm{m}$ intervals along the longitudinal direction of the specimen, and the deformed shape of the specimen could be easily identified. Using this sensor, the alignment between the specimen and the loading tip can be checked and adjusted using the five-axis stage of the specimen holder, and the deflection angle $\theta$ of the specimen can be readily obtained from the deformed shape.

\subsection{Test procedure}

It is very important to align the loading tip, displacement sensor, and specimen since misalignment among them can cause errors in the estimation of elastic modulus and yield strength. The movement of the loading tip and the deflection of the specimen can be accurately monitored by the laser displacement sensor, and the misalignment is 

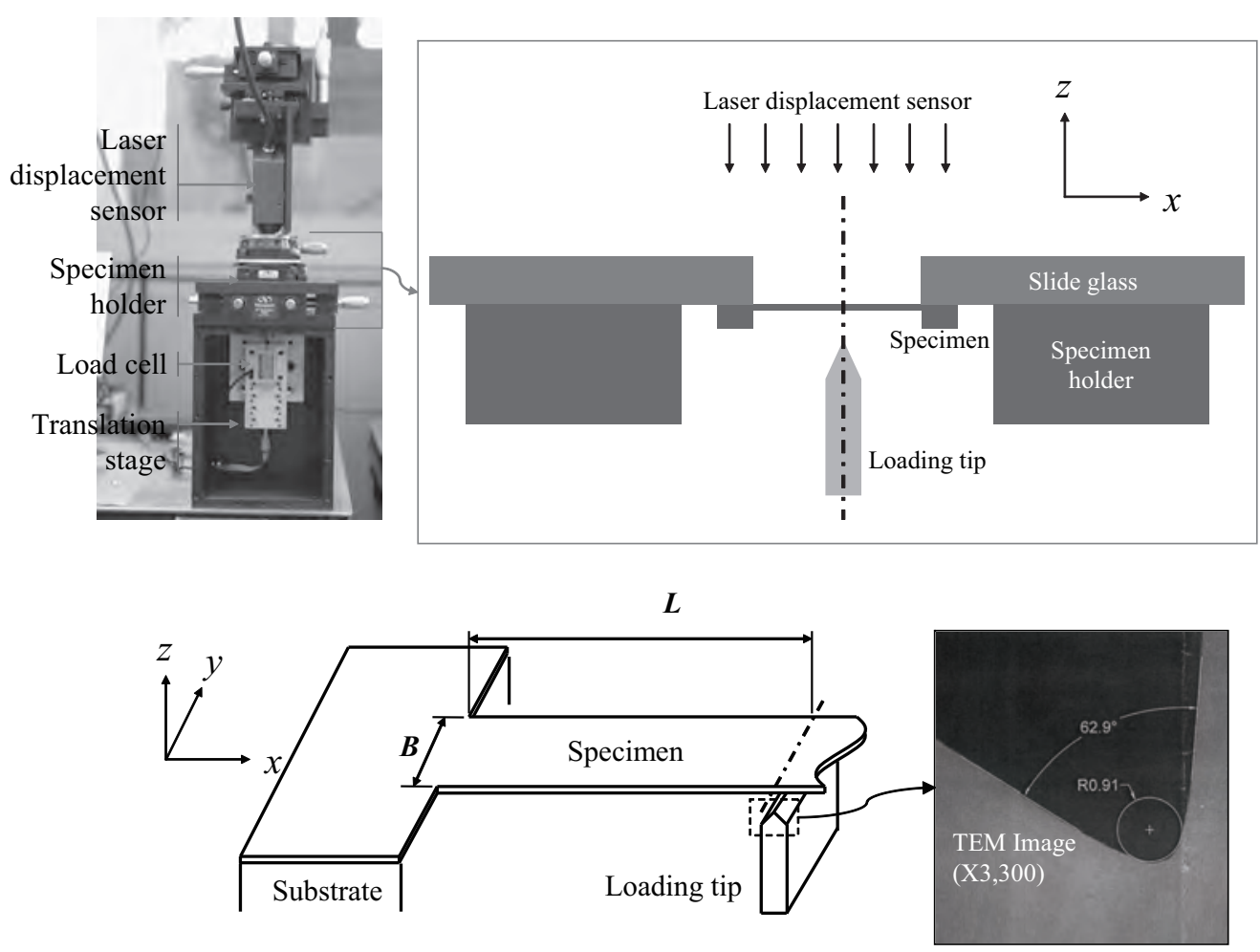

Fig. 4. Photograph of strip bending apparatus.

markedly reduced by adjusting the five-axis stage of the specimen holder as follows: (1) the laser displacement sensor is carefully aligned perpendicular to the moving axis (z-axis) of the loading tip by adjusting the fixture holding the sensor. (2) The strip specimen bonded to a transparent glass slide is then attached to the specimen holder (see Fig. 4). (3) The specimen is aligned to the sensing axis ( $z$-axis) of the laser displacement sensor by adjusting the rotations of the specimen holder in the $x$-, $y$-, and $z$-axes. (4) Finally, the specimen holder is moved in the $x$ - and $y$-axes to align the center of the specimen right above the loading tip.

When the above alignment procedure is completed, the strip specimen is deflected by moving the motorized stage holding the loading tip, and the load-displacement data is measured. The displacement sensor adopted in this study measures the deflected shape by scanning a line on the specimen, and requires approximately $1 \mathrm{~s}$ to complete a single line scan. During this period, the specimen should be stationary, making it difficult to test the strain-rate dependency of materials. The typical history of the displacement of the motorized stage during the test is shown in Fig. 5. When the deflection shape of a specimen is fully understood, no line scan is necessary and only a single point measurement of the deflection is sufficient. In this case, test speed can be greatly improved. 


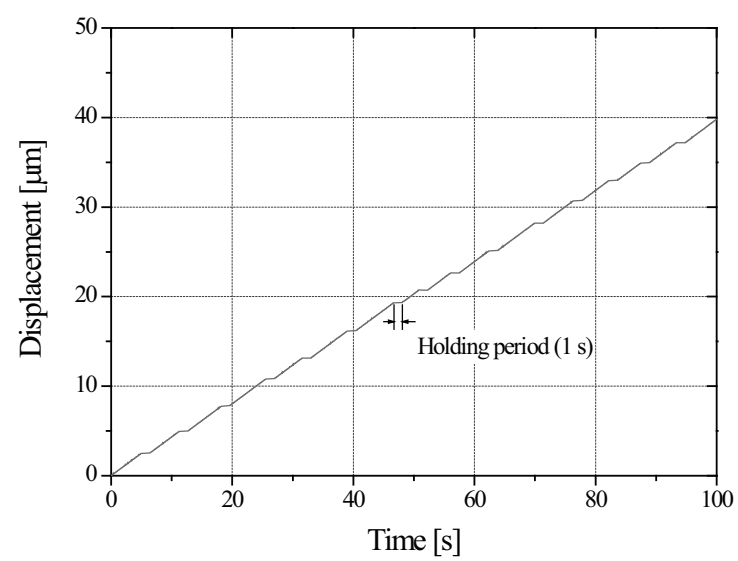

Fig. 5. Typical displacement history of strip bending test.

\section{Results and Discussion}

\subsection{Strip bending test results}

The deflection shapes of the two types of strip specimen are shown depending on the load in Fig. 6. The corresponding stress-strain curves are obtained from these data using eqs. (3) and (4), and are shown in Fig. 7. All the specimens were initially buckled, and this means that the specimens were initially under compressive stress. The elastic modulus of the specimens was determined by fitting the stress-strain curve from 100 to $200 \mathrm{MPa}$. The stress-strain curves of both types of specimen were similar to each other, but the elastic modulus from the curved specimen was slightly higher than that from the straight one, as shown in Table 1.

The measured elastic modulus is lower than that $(77.2 \mathrm{GPa})$ of bulk gold. ${ }^{(12)}$ This can be explained from the difference in microstructure between the present Au thin film and bulk gold. The average grain size of the present Au thin-film specimen is approximately $98.2 \mathrm{~nm}$, as described in $\S 3.1$. The small grains can lead to a high volume fraction of grain boundary, and can result in low modulus. ${ }^{(13)}$ For this reason, the present $\mathrm{Au}$ specimen with nanoscale grains can have lower modulus than bulk Au material with microscale grains. The yield strength $(383 \mathrm{MPa})$ of the present $\mathrm{Au}$ specimen is higher than that $(120 \mathrm{MPa})$ of the bulk, ${ }^{(12)}$ and this can be explained in terms of grain size difference. Materials with smaller grains are usually stronger than those with larger grains, and this can be characterized by the Hall-Petch relation. ${ }^{(14)}$

\subsection{Discussion and comparison with tensile test results}

The strip bending test can measure the stress-strain curve similarly to the microtensile test. It is appropriate to perform the microtensile test on a Au freestanding film fabricated using the same process as the strip bending specimen. For this purpose, we 
(a)

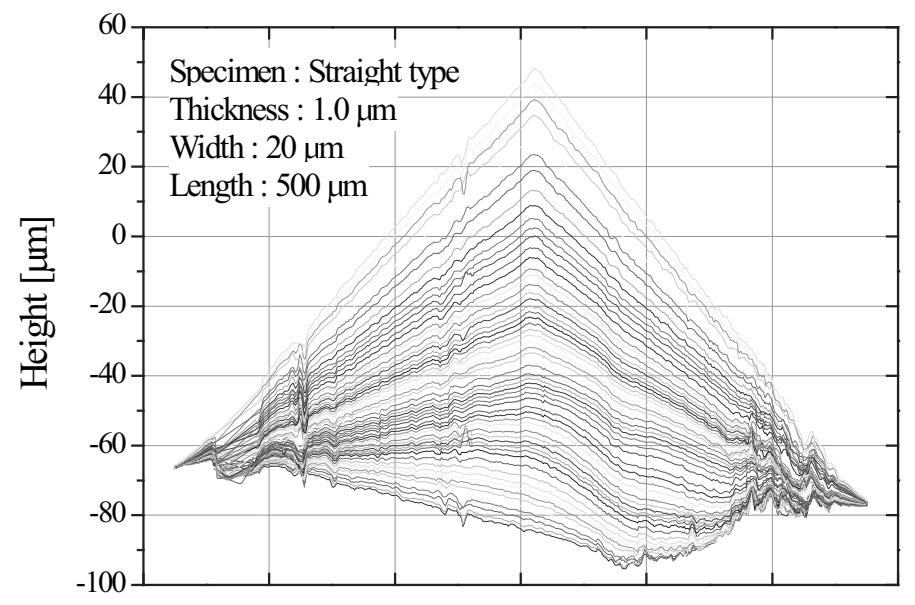

(b)

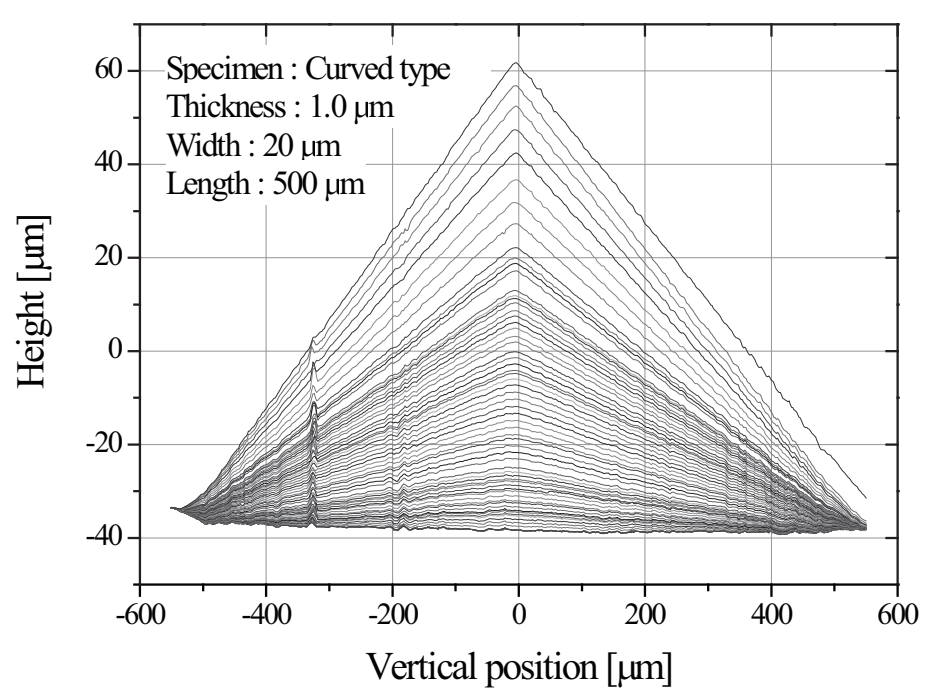

Fig. 6. Deflection shapes of (a) straight and (b) curved specimens.

fabricated microtensile specimens together with strip bending specimens on a single $\mathrm{Si}$ wafer; this was easily achieved by designing a mask containing both the microtensile specimens and the strip bending specimens. We have already reported an accurate microtensile testing apparatus that incorporates alignment stages, a sensitive load cell, a piezoelectric actuator, and a noncontact strain measurement module based on digital image correlation. ${ }^{(4)}$ Using this apparatus, the tensile stress-strain curve of a $\mathrm{Au}$ freestanding thin film was measured, as shown in Fig. 7. The stress-strain curves from the strip bending test are also plotted in the figure for comparison. Curves from the strip 


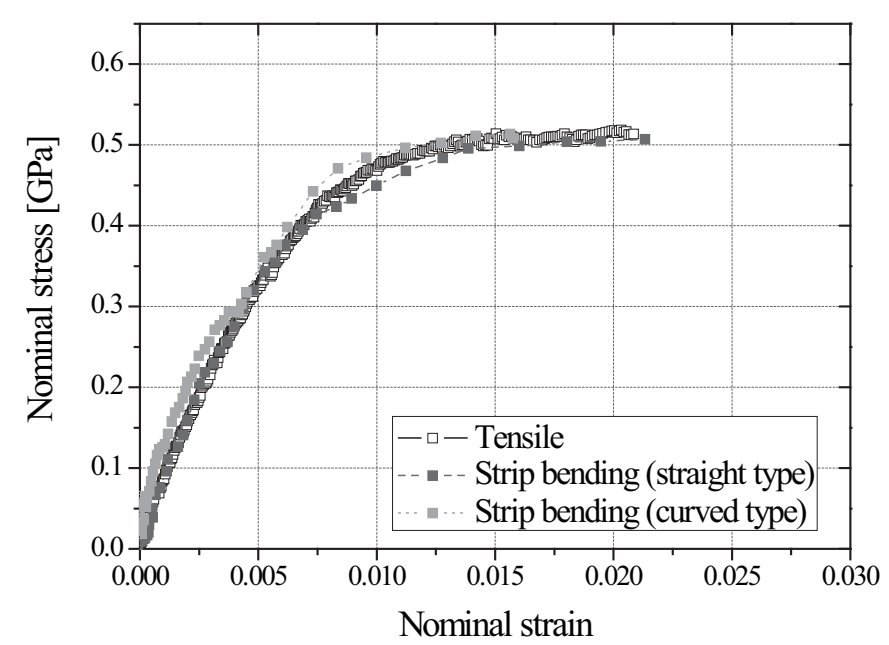

Fig. 7. Comparison between strip bending and microtensile test results.

Table 1

Comparison of elastic moduli measured by strip bending and microtensile tests.

\begin{tabular}{lccc}
\hline & Specimen type & \multicolumn{2}{c}{ Elastic modulus (GPa) } \\
\cline { 3 - 4 } & & Average & Standard derivation \\
\hline Tensile test & Dog-bone & 60.4 & 1.5 \\
Strip bending test $\begin{array}{c}(w=200 \mu \mathrm{m}, t=1 \mu \mathrm{m}) \\
\quad \text { Straight } \\
(w=200 \mu \mathrm{m}, t=1 \mu \mathrm{m}) \\
\quad \text { Curved }\end{array}$ & 63.5 & 8.8 \\
& & 73.8 & 12.1 \\
$(w=200 \mu \mathrm{m}, t=1 \mu \mathrm{m})$ & & \\
\hline
\end{tabular}

specimens can overlap onto tensile data by shifting along the horizontal axis owing to the initial strain in strip specimens.

\subsection{Discussion and comparison with finite element analysis}

Finite element analysis was performed in order to understand the effect of specimen shape on the test results. Three-dimensional finite element models for the two types of specimen were developed (see Fig. 8) and their deformation behaviors were simulated using a commercial finite element package, ABAQUS. ${ }^{(10)}$ The convergence, verification, and other simulation details of the finite element analysis will be presented in another paper. $^{(11)}$

The elastoplastic deformation of the two types of strip specimen was simulated using the input parameters (i.e., elastic modulus and flow law) measured by micro-tensile test, except for Poisson's ratio. Poisson's ratio was assumed to be 0.42 , which is that of bulk Au. ${ }^{(12)}$ As can be seen in Fig. 8, the strain distribution is quite uniform along 

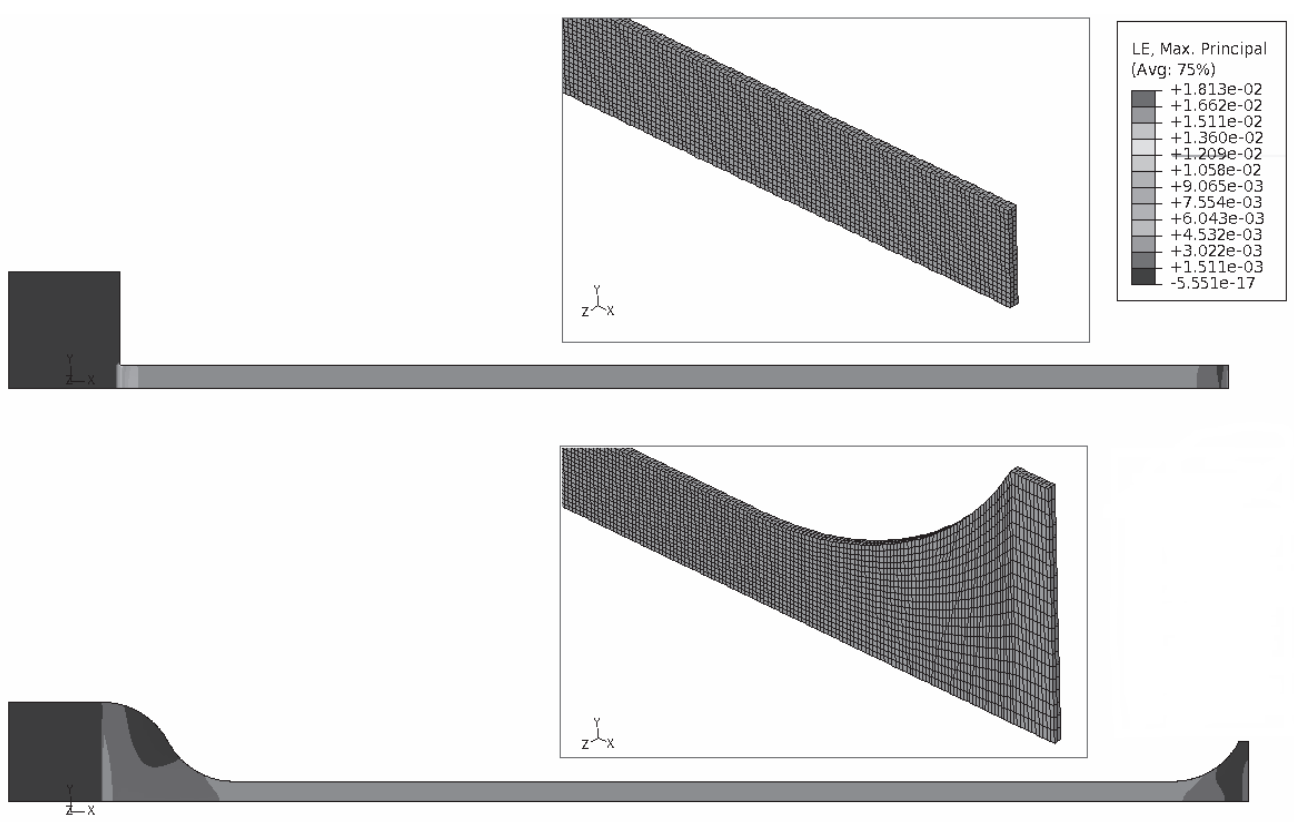

Fig. 8. Finite element mesh and strain distribution for straight and curved specimens.

the whole length of the straight specimen except at the fixed and loaded ends, while the region of uniform strain is much reduced in the curved specimen. This finding indicates that the analysis model in $\S 2.3$ is more appropriate for the straight specimen than for the curved specimen since the model relies on the assumptions of uniform strain along the specimen. In the simulation, the input value for the elastic modulus parameter was $60.4 \mathrm{GPa}$; simulated elastic modulus values were $60.8 \mathrm{GPa}$ for the straight specimen and $66.9 \mathrm{GPa}$ for the curved specimen. The finite element analysis predicted that the difference in elastic modulus should be negligible for the straight specimen, but not for the curved one. The experimental results of the strip bending test were consistent with the simulation results in that elastic modulus values were closer to those measured by microtensile testing in straight specimens than in curved specimens.

\subsection{Desirable test practice for strip bending method}

As with other test methods, desirable test practices for the strip bending method should be established. The apparatus for strip bending should incorporate suitable components. One of the key components is the adjustment stage for alignment, since the test results are sensitive to alignment. The effect of misalignment on the test results is an interesting topic that we deal with in another paper. ${ }^{(10)}$ The displacement and load sensors should have resolutions better than 1/1000 of the maximum deflection and load. Instead of a displacement sensor of a single-point measurement, it is useful to adopt a displacement sensor that can measure deflection distribution for verification purposes 
similarly to this study. For actuating devices, all types of microscale actuator can be used as long as a linear movement of the loading tip is guaranteed. It is desirable to use a wedge-shaped tip for loading to apply a line contact load to the strip specimen. The material of the tip should be rigid, and diamond and sapphire are good candidates. The root radius of the tip should be carefully chosen depending on the specimen size. The contact area between the tip and the specimen increases with the tip radius, and this can result in errors of the measured properties. On the basis of the finite element simulation results, ${ }^{(11)}$ the tip radius should be less than $1 / 50$ of the half-length of the specimen. The lower bound of the tip radius has not been studied yet.

As shown in the previous sections, the specimen geometry should be carefully chosen. If one wants to measure the elastic modulus of thin films, a straight specimen will be beneficial. For yield strength measurement, the two types of specimen have their respective disadvantages. The straight specimen suffers from strain and stress concentration while the curved one suffers from low accuracy of strain measurement. For tensile strength measurement, the curved one will be beneficial. Depending on the purpose of the test, the specimen shape should be carefully chosen.

For the testing procedure, the procedure for alignment is critical and one needs to minimize the misalignment using the adjustment stage. Testing speed is another key issue in the strip bending test. A constant speed of the loading tip can be easily achieved by controlling the actuator, but a constant speed of the loading tip does not lead to a constant strain rate in the strip specimen, as can be seen in eq. (2). Controlling the actuator to obtain a constant strain rate in the specimen appears to be difficult, but can be realized using a sophisticated control algorithm for the actuator. This topic is beyond the scope of this paper. We have studied a control algorithm for strain-rate-controlled strip bending testing of polymeric thin film and will describe this algorithm and its results in a forthcoming paper.

\section{Summary}

The strip bending method was applied to the measurement of the stress-strain curves of Au thin films fabricated using the MEMS process. The effect of specimen shape on the test results was investigated using experiments and numerical simulation. The test results obtained from two types of strip specimen were compared with those measured by the microtensile test. Straight specimens provided results closer to those of microtensile testing than curved specimens, and finite element analysis revealed that this difference was due to differing strain distributions between the two types of specimen. A desirable test practice of the strip bending method was suggested for standardization purposes on the basis of the experimental and FEA results. This work demonstrated experimentally and numerically that the strip bending test could produce stress-strain data of freestanding thin films cost-effectively, and that the consistency of its results with the tensile test results could be increased by proper design and practice of the strip bending test. 


\section{Acknowledgement}

This research was supported by grant 08-K1401-00610 from the Center for Nanoscale Mechatronics \& Manufacturing, a 21st Century Frontier Research Program supported by the Korean Ministry of Education, Science and Technology.

\section{References}

1 M. Gad-el-Hak (ed.): The MEMS Handbook (CRC Press, New York, 2001).

2 W. D. Nix WD: Metall. Trans. A 20 (1989) 2217.

3 W. N. Sharpe: Exp. Mech. 43 (2003) 228.

4 S. J. Lee, S. M. Hyun, S. W. Han, H. J. Lee, J. H. Kim and Y. I. Kim: Adv. Mater. Res. 26-28 (2007) 1117.

5 H. D. Espinosa, B. C. Prorok and M. Fisher: J. Mech. Phys. Solids 51 (2003) 47.

6 C. W. Baek, J. M. Kim, Y. K. Kim, J. H. Kim, H. J. Lee and S. W. Han: Sens. Mater. 17 (2005) 277.

7 J. H. Kim, H. J. Lee, S. W. Han, J.Y. Kim, J. S. Kim, J. Y. Kang, S. H. Choa and C. S. Lee: Int. J. Mod. Phys. B 20 (2006) 3757.

8 J. H. Kim, H. J. Lee, S. W. Han, J. M. Kim and C. W. Baek: Key Eng. Mat. 321-323 (2006) 121.

9 D. I. Kim, Y. H. Huh, D. J. Kim, Y. H. Lee and C. D. Kee: J. Mater. Process. Tech. 187,188 (2007) 232.

10 www.simulia.com

11 J. Park, J.-H. Kim, H.-J. Lee and B.-I. Choi: in preparation for publication.

12 www.matweb.com

13 H. S. Kim and M. B. Bush: Nanostruct. Mater. 11 (1999) 361.

14 C. S. Pande and K. P. Cooper: Prog. Mater. Sci. 54 (2009) 689. 